\title{
ENDOCERVICOSIS VESICAL: EXCEPCIONAL CAUSA DE MASA VESICAL
}

\author{
C. GONZÁLEZ-SATUÉ, J. SERRA CÁNER, J. ARMORA MANI \\ Servicio de Urología. Hospital Sant Jaume de Calella. Consorci Hospitalari de la Selva i el Maresme. \\ Barcelona.
}

Actas Urol Esp. 28 (9): 677-679, 2004

\section{RESUMEN}

ENDOCERVICOSIS VESICAL: EXCEPCIONAL CAUSA DE MASA VESICAL

Describimos un caso clínico de una mujer de 32 años con endocervicosos vesical y endometriosis ovárica. La asociación de endocervicosis con endometriosis es posible debido al origen mülleriano de ambos procesos. La endocervicosis vesical es una entidad excepcional que puede presentarse en mujeres jóvenes como una masa vesical simulando un cáncer vesical. Revisamos los casos previamente publicados en la literatura.

PALABRAS CLAVE: Endocervicosis. Masa vesical. Endometriosis ovárica.

\section{ABSTRACT}

BLADDER ENDOCERVICOSIS: AN EXCEPTIONAL CAUSE OF BLADDER MASS

We report a case of a 32 year-old woman with bladder endocervicosis and ovarian endometriosis. Association of endocervicosis with endometriosis is feasible due to a müllerian origin of both entitties. Bladder endocervicosis is an exceptional entity that can be present in young women as a bladder mass like a bladder cancer. We review previously published cases in literature.

KEY WORDS: Endocervicosis. Bladder cancer. Ovarian endometriosis.

$\mathrm{S}_{\mathrm{s}}^{\mathrm{c}}$ conoce por endocervicosis a la presencia de ormaciones glandulares del cérvix uterino fuera del canal endocervical. Se trata de una patología benigna, que puede presentarse asociada a otros procesos en los que prolifera tejido de origen mülleriano como la endometriosis, o la endosalpingosis. Su aparición en vejiga es excepcional y, al contrario que la endometriosis presenta una clínica inespecífica, aunque igualmente con oscilaciones menstruales. Su hallazgo en la vejiga es extremadamente infrecuente y puede confundirse con un cáncer vesical. Describimos a continuación el caso clínico de una endocervicosis vesical que fue etiquetada inicialmente como un carcinoma vesical transicional y que fue tratada de forma satisfactoria con resección transuretral.

\section{CASO CLÍNICO}

Mujer de 32 años, con antecedentes patológicos de síndrome depresivo y dismenorrea. Consulta por urgencia miccional, incontinencia de urgencia y polaquiuria diurna y nocturna de aproximadamente dos años de evolución, la clínica se exacerba con la menstruación y niega la aparición de hematuria macroscópica. Los urocultivos realizados en el centro de asistencia primaria han sido repetidamente negativos, en el sedimento aparece leucocituria discreta y el tratamiento antibiótico empírico a la que se ha sometido no mejora la clínica miccional.

Ya en la consulta urológica se realiza análisis sanguíneo, que es estrictamente normal, una flujometría urinaria que muestra un flujo máximo 
de $30 \mathrm{ml} / \mathrm{seg}$ y un flujo medio de $15,1 \mathrm{ml} / \mathrm{seg}$ para un volumen miccional de $422 \mathrm{ml}$ con una curva de morfología normal. Se practica una ecografía renovesical en la que destaca la presencia de una masa endovesical de $41 \times 26 \mathrm{~mm}$, ubicada en suelo vesical y de aspecto infiltrante de la musculatura.

Bajo la sospecha de tumor vesical transicional no se solicitan citologías y se procede a resección transuretral. En la cistoscopia se aprecia una masa de crecimiento submucoso, con muscular indemne. Al corte se evidencian pequeñas colecciones de aspecto quístico, con contenido achocolatado, y en ocasiones mucoso, en su interior, se aprecia invasión macroscópica de la musculatura vesical.

El diagnóstico anatomopatológico del material remitido es compatible con glándulas de cervix uterino secretoras de moco, proceso sugestivo de endocervicosis vesical. No se evidencian signos de malignidad.

La paciente queda asintomática desde el punto de vista urológico, por lo que sigue controles posteriores por Ginecología. Se realiza TC abdominal (Fig. 1), el que se observa endometrioma ovárico izquierdo, por lo que se somete a una anexectomía izquierda laparoscópica y tratamiento con análogos de la LH-RH. Actualmente, después de 16 meses de seguimiento tras la RTU, la paciente se encuentra asintomática y sin recidiva.

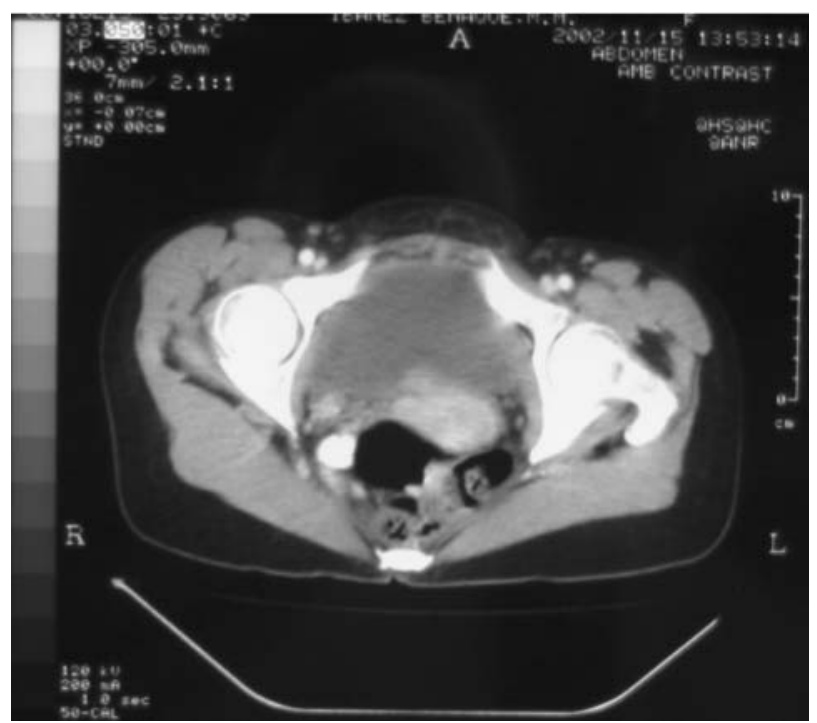

FIGURA 1. TC abdominal mostrando masa ovárica izquierda (endometriosis ovárica).

\section{DISCUSIÓN}

Se conoce por endocervicosis la presencia de tejido con glándulas endocervicales fuera de su lugar anatómico habitual: el canal endocervical. Fue descrita por primera vez por Goodall y cols. ${ }^{1}$ en 1943 como una lesión infiltrante de tejidos pélvicos paracervicales constituido por una proliferación benigna de glándulas endocervicales. Otras ubicaciones donde ha sido descrita la endocervicosis han sido el peritoneo pélvico y los ganglios linfáticos, en estos casos el diagnóstico suele ser incidental ${ }^{3}$.

La endocervicosis vesical es una entidad excepcional, sólo se han descrito previamente unos 13 casos en la literatura ${ }^{2-9}$, y sólo uno de ellos en nuestro país ${ }^{9}$. Los primeros en descibirlo en la vejiga fueron Clement y Young ${ }^{2}$, en 1992, que recogieron 6 casos, todos ellos eran masas de entre 2 y 5 centímetros, localizadas en la base o cara posterior de la vejiga, de aspecto macroscópico infiltrante que obligó a cistectomía parcial en 5 de los 6 casos, el otro pudo ser controlado con resección transuretral.

La endocervicosis es una entidad clínica relacionada con la endosalpingisosis y con la endometriosis y con las que tiene un origen embriológico común: diferenciación no maligna del llamado sistema mülleriano secundario, procedente de la cavidad celómica del embrión ${ }^{9}$ y que se diferenciará posteriormente en endometrio, endocervix o tejido tubárico, por lo que su aparición conjunta no es excepcional, aunque no es frecuente, sólo en 3 de los trece casos descritos había antecedentes de endometriosis ${ }^{3,6,9}$. Nunca ha sido diagnosticada en hombres y no se conoce transformación maligna. La edad de aparición es la época fértil de la vida, predominantemente en la cuarta década.

La presentación clínica suele ser inespecífica, en forma de polaquiuria, frecuencia miccional, dolor pélvico, e incluso síntomas obstructivos o hematuria. A diferencia de la endometriosis vesical, la aparición de hematuria no es la norma. Los cultivos y las citologías son negativos y el diagnostico se realiza por ecografia, aunque la confirmación solo puede realizarse con el examen anatomopatológico de la muestra quirúrgica.

El diagnóstico diferencial debe hacerse principalmente con el adenocarcinoma vesical y el 
carcinoma de células transicionales. Aunque se trata de un proceso benigno, el aspecto macroscópico infiltrante y sus similitudes histológicas con el adenocarcinoma pueden llevar a un diagnóstico erróneo ${ }^{2}$. Por este motivo ha sido tratada principalmente con cistectomía parcial ${ }^{2,9}$, e incluso se ha requerido el tratamiento laparoscópico $^{5}$, para conseguir la exéresis completa, pero la resección transuretral se ha mostrado suficiente para el correcto control del proceso ${ }^{2,6}$. No se ha descrito ningún caso de recurrencia o de malignización a pesar de haber seguimientos de hasta 14 años.

Julie y cols. ${ }^{7}$ hacen un estudio inmunohistoquímico de un caso de endocervicosis y lo comparan con tejido endocervical normal, encuentran mayor expresión de receptores estrogénicos y de progesterona, de ello se induce el uso de tratamiento hormonal en la resecciones parciales. También encuentran un índice mitótico superior en la endocervicosis comparado con el cervix normal, pero dentro de los límites considerados como endocervix no patológico.

\section{CONCLUSIÓN}

La endocervicosis vesical en una infrecuente entidad que se presenta en mujeres de edad fértil, de carácter benigno, aunque la pruebas de diagnóstico por la imagen puedan mostrar un aspecto infiltrante que pueda sugerir un carcinoma. En casos de tamaño adecuado se puede conseguir un control endoscópico de la enfermedad, junto con tratamiento hormonales. Creemos que la cistectomía parcial estaría indicada para casos extensos o para recurrencia de síntomas, proceso que aún no ha sido descrito en la literatura.

\section{REFERENCIAS}

1. Goodall JR. A Study of Endometriosis, Endosalpyngosis, Endocervicosis and Peritoneo-Ovarian sclerosis. Philadelphia: J.B. Lippincott Co 1943:89.

2. Clement PB, Young RH. Endocervicosis of the urinary bladder. A report of 6 cases of benign mullerian lesion that may mimic adenocarcinoma. Am J Surg Pathol; 16(6):533-542.

3. Parivar F, Bolton D, Stoller M. Endocervicosis of the bladder. J Urol 1995;153:1218-1219.

4. Rodriguez R, Alfert H. Endocervicosis of the bladder: a rare mucinous analogue of endometriosis. J Urol 1997;157:1355.

5. Nada W, Parker J, Wong F, Cooper M, Reid G. Laparoscpic excision of endocervicosis of the urinary bladder. J Am Assoc Gynecol Laparoscop 2000;7:135-137.

6. Spencer S, Rubin M, Hussain H, Wolf S Jr. Complete transuretral ressection of bladder endocervicosis. J Urol 2001;164:524.

7. Julié C, Boyé K, Desgrippes A, Régnier A, Staroz F, Fontaine E, Franc E. Endocervicosis of the urinary bladder. immunohistochemical comparative study between a new case and normal uterine. Pathol Res Pract 2002;198:115-120.

8. Seman EI, Steward TJ. Endocervicosis of the urinary bladder. Austr New Zeal J Obst Gynaec 1994;34:496.

9. Leiva O, Ortiz Vico F. Endocervicosis de la vía urinaria: sistema mülleriano secundario. Patologías excepcionales en Urología. Ed. Luzán 1998:11-43.

Dr. C. González-Satué

C/ Monte, 95 - Esc. 6, $1^{\circ} 2^{\mathrm{a}}$

08340 Vilassar de Mar (Barcelona)

(Trabajo recibido el 1 diciembre de 2003) 